\title{
Foundation-phase children's causal reasoning in astronomy, biology, chemistry and physics
}

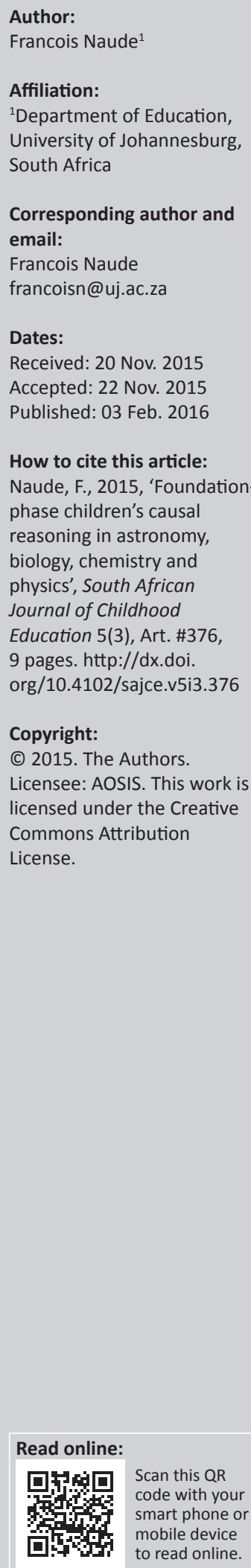

\begin{abstract}
This study aimed at finding out how young children express their thinking about some of the themes in the early-grades school science curriculum. Foundation-phase children at a primary school in Soweto, Johannesburg, were interviewed after they had watched classroom demonstrations of the production of carbon dioxide gas, absorption of water and the propulsion of an inflated balloon and after a discussion about living organisms had been introduced. Interviews were conducted in each of four classes, ranging from grade $\mathrm{R}$ to grade 3, asking questions about what they had just observed in the four different lessons. The findings show that the children expressed their own ideas, but that they were using the discourse structure of causality, albeit that the content of the reasoning came from their naïve or intuitive theories. The article recommends that teachers in the primary school consider (and utilise) children's spontaneous reasoning and naïve or intuitive theories of natural phenomena when they teach the natural science curriculum. This study has shown that, although early-grades children may lack formal science concepts, their ability to reason is developed sufficiently to grasp the causal relationships in natural phenomena. The question this research asks, ultimately, is, 'How can some basic science concepts be woven into the curriculum of the entire foundation phase curriculum, integrating it with language and literacy, and especially with mathematics?' The author argues that firmer conceptual knowledge of science can be developed in younger children in the early grades, because the thinking mechanisms for understanding and reasoning seem to be in place at a young age.
\end{abstract}

\section{Introduction: Laying the foundation for science concept development}

Before children reach the formal science class in the upper grades of the primary school (known as 'the intermediate phase' in South Africa), they are already familiar with many ideas about science and have developed what various authors describe as 'naïve' concepts (Spelke 2010) or 'intuitive' theories (Carey 2009; Pine, Messer \& St. John 2001). Their experience of interacting with the natural world, as well as their sociocultural world, facilitates the formation of naïve concepts, based on 'intuitive theories'. Carey (2009) and Gopnik (2011) describe the formation of such theories through the lens of the so-named theory theory of conceptual development (Henning \& Ragpot 2015), meaning that children are like 'little scientists', using their own methods of observation, hypothesising and testing. Vosniadou and Ioannides (1998) argue that one of the most important findings from cognitive developmental research is that children do not enter the science class room as a blank slate, but rather, as a 'messy slate', because of their everyday interaction with the natural world and, I would add, in which they have formed their own working theories. By this way, they build rich personal knowledge. Examples of how children learn the effects of certain actions in the physical world, such as falling down a staircase when moving too fast, getting burnt when touching the flame of a candle, making a 'mess' when a container filled with liquid falls to the ground, are many. At the same time, because they live, also, in a sociocultural setting, where language is learned spontaneously, they chance to know the linguistic discourse markers of causality, such as, 'if that happens, then it is likely that this will follow'. This linguistic structure is, according to some of the authors who study children's development (Gopnik 2003; Gopnik et al. 2001), specifically their development of language with which to make their way in the sociocultural world, which is one scaffolding framework for causal reasoning. Children hear the reasoning and they speak it. Vygotsky and Luria (1994) addressed this issue extensively in the 1930s and attribute 'higher psychological functions', such as causal reasoning, to the role of 'sign organisation', specifically language as sign. Jean Piaget and his research collaborators argued in a number of publications that logic is inherent in child development (Piaget \& Inhelder 1969; Piaget \& Inhelder 1975; Piaget, Inhelder \& Häfliger 1977), and Vygotsky (1986) titled one of his two primary works Thought and Language. Rogoff (1990), working in the Vygotskian tradition, argues that cultural apprenticeship also means linguistic and cognitive apprenticeship. This view was 
also developed by Jean Lave (1988), and John Seely Brown and his colleagues (Brown, Collins \& Duguid 1989).

This study set out to locate children's expression of their causal reasoning, asking the question: 'How do children in the foundation phase respond to science demonstrations and a discussion in terms of their causal reasoning about the phenomenon or the objects which they had just witnessed and about which they had been talking?'

\section{The science curriculum in the primary school}

When children in South African schools enter the intermediate phase, they engage with a demanding science curriculum. The four strands of the curriculum are life and living; energy and change; matter and material; and earth and beyond (South Africa. Department of Basic Education 2011). Topics from the domains of biology, chemistry, physics and astronomy are therefore formally taught in this phase, expecting children to learn through language, pictures and (only sometimes) through conducting 'experiments' or going on an outing where they can get in touch with, especially, the living world in a nature setting, as described by Klaar and Öhman (2014) and Passy (2014). The foundation-phase curriculum, however, does not require science teaching in the same depth and detail as the intermediate phase and children are largely left to the development of their spontaneous development, based on some ad hoc instruction. Some concepts from biology are taught in the foundation phase, but very few concepts from chemistry, physics or astronomy are included in the general learning area of 'life skills', which is an umbrella term for all learning activities that are not in the language and literacy or the mathematics curriculum. This is also evident in the study by Saçkes (2014:171), who argues that: (a)lthough science content standards provide clear expectations for teachers of young children about the science concepts they are supposed to teach, no studies have been conducted to examine how often standard-based science concepts are being taught in kindergarten classrooms and the factors that influence the frequency of science instruction.

\section{Saçkes (2014) also says that:}

(r)ecent literature suggest that early childhood teachers feel pressured to devote most of their instructional time to the teaching of language and literacy (Greenfield et al. 2009). Teachers with greater control over their curriculum might be more likely to teach science than peers who believe that they have little control over the curriculum. Thus, teachers' perceptions of control over the curriculum (teachers' perceptions) were hypothesized to influence the teaching of science concepts.

This statement holds much truth for the South African school curriculum as I have observed it in action at schools. But, in addition to lack of studies on young children's science learning, and control of the curriculum, there is, according to Saçkes, also the issue of teachers' content knowledge of science: Saçkes (2014:181) concludes that one of the findings of his study 'suggests that other factors, such as limited content knowledge and low efficacy for teaching'.
Saçkes (2014:181) tested a theoretical model of science teaching in kindergarten with public data $(n=3305)$ and found that:

(t)he number of science methods courses that teachers previously completed was a statistically significant predictor of the frequency of teachers' teaching of life science, which was the most prominent area of science taught according to this inquiry. This leaves the children of the study I have analysed as example in more or less the same situation and the children that I am studying.

In effect, most children in the foundation-phase classrooms that I study in South Africa do not have a foundation on which to build the many new facts and concepts that they are expected to learn from grade 4 onwards. One way of addressing this issue of curricular constraints in the foundation phase is by crossing the current very firm subject area boundaries, in other words, by inserting science into both the mathematics and the language curriculum. Such an integrated approach would mean that mathematics is placed in the real, physical world, which does not mean it is concretised by using material objects / manipulatives only, but by making maths learning a living experience of the physical world: this living experience can then be internalised mentally by learning to use symbols with which to represent the physical world (Bowerman \& Levinson 2001). And so, the numeral, 'four' is internalised as a linguistic symbol for the quantity, whilst the Arabic notation, ' 4 ', is internalised in the same way as the linguistic term, and thereby creates a parallel representation, whilst the four concrete objects, like four leaves/blocks, stones/marbles, are the fixture in the physical world. Because concept development and reasoning, including causal reasoning (Legare \& Clegg 2015) are abstract formations (or 'higher psychological functions', according to Vygotsky 1978), they build on each other in 'mental representations' (Carey 2009) where neurological connections physiologically make connections and where a whole world of representations develop, with the 'symbols serving as shortcuts' (or 'placeholders', according to Carey 2009). Writing an equation, such as $4+6=10$, is then an externalisation of a mental representation, as is 'six more than four adds up to ten'. These externalisations are then symbolic representations of what is known non-symbolically (Carey 2009). Carey calls the real-world (non-symbolic) objects 'core domains' and the mental (symbolic) representations 'core cognition'. Both symbols and concepts are important developmental indicators.

The argument, or the hypothesis, of this article is that much of what is learned in physics (and the other strands in the science curriculum as well) can be synthesised in the language and the mathematics curriculum of the early grades. The instances where language and literacy feature in mathematics and science are many: Take, for example the teaching of the structures of the language of maths. Henning (2012) argues that learning in the early grades is learning in a 'linguistic maze'; children negotiate morphemes (especially in the agglutinative languages of SA), words and phrases that will be remembered after they have been processed 
in the working memory (Cockcroft 2015). These will be retrieved from memory when needed as maths 'facts' and used to try to make sense of maths problems. Many of these are formulated in language and if the language structures and the vocabulary are not understood, language (linguistic representation) becomes the obstacle in mathematics problem solving, rather than maths itself being the problem. I would argue that the same accounts for physics and that these two 'learning areas' can comfortably be combined, so that the physics concepts and facts can scaffold the mathematical concepts and facts and vice versa, with language as the mediating set of symbolic representation during interaction with teachers and peers - much as Vygotsky had intended it in his theorising of semiotic mediation (Henning 2012; Henning \& Dampier 2012).

For example, calculating the velocity of an object requires both mathematical concepts and physics concepts operating in tandem in a young learner's mind. Illustrating/ demonstrating/operationalising these concepts for children is not difficult. They may be able to calculate the velocity of a ball thrown between two points by measuring the distance between the two points and dividing it by the time it takes the ball to travel between the two points. In this instance, a concept from physics is used to scaffold the mathematics concept and concomitant operations of measurement and division. This example can be amended to include two balls traveling at different velocities and calculating the time it would take for each ball to travel the same distance. Mathematical procedures are therefore used to calculate a physics phenomenon. The benefit of combining physics and mathematics concepts, as in this example, is that concepts from both 'learning areas' are made concrete and are 'in operation' (Furner \& Kumar 2007). In the higher grades, this type of integration is expected to be firmly established.

I thus argue in this article that an integrated curriculum of science and mathematics in the early grades may be a way to advance learners' knowledge in both these areas and, importantly, make use of their 'proclivity to reason causally', whilst scaffolding their knowledge to do so with 'suitable content'. And this, I would argue, is part of the brief of a teacher. Teachers can assist in ordering the intuitive theories that children hold into an accurate coherent set of conceptual systems, or collections/networks of concepts (Carey 2009; Henning 2013) whilst also drawing on their (childlike) reasoning skills to organise the facts and concepts. A prudent teacher would not want to lose the opportunity of furthering reasoning skills with factual/conceptual knowledge that is 'not' usable; in other words, teachers would want to assist children to move from their intuitive theories to conventional theories and facts of science phenomena, instead of using ad hoc examples that do not cohere (such as the designers of tests often do - using oranges and apples and other objects and phenomena as examples that do not cohere materially or conceptually). An integrated curriculum could consist of a systematic use of science content knowledge to form the object world of mathematics problems. Instead of using arbitrary examples when doing math problems, teachers can use examples from everyday physics that relate to children's experiences.

In order for this to occur in the mind of the learning foundation-phase child, conceptual change has to occur; young children have to come to grips with the 'scientific' meaning of concepts by gradually changing 'spontaneous' concepts (as Vygotsky [1978] described the development of 'higher psychological functions'). Before teachers can even attempt to start teaching a new concept (to utilise in reasoning, going beyond factual knowledge), they need to determine what the intuitive theories are that a child holds. In the next section of the article, I will discuss the notion of intuitive theories of children, specifically as propounded by Carey (2009).

I will then describe the research project on which this article is based and explain the findings of the research. I will conclude the article by discussing the implications these findings may hold for the teaching of science in the foundation phase, accentuating causal reasoning.

\section{Children's theories of the natural world}

Viewed from a constructivist epistemology, primarily as set out by Piaget (1952), it is important for a teacher to establish which foundation (no matter how 'unscientific' it might be) may have been laid by children's interactions with the environment, before attempting to build on this foundation with the introduction of new concepts. A myriad of disciplines have long attempted to identify ways in which one can find out what and how children think and conceptualise. These disciplines stretch through the academic spectrum from philosophy and developmental psychology to neural imaging techniques and standardised testing (Gopnik, Meltzhof \& Bryant 1997; Henning \& Ragpot 2015).

I would argue that science teachers and educational researchers concerned with studying science education need to know what children know, before they can even attempt to teach them. If this is not achieved, teachers will not know what content and skills to teach and even more important, how to teach these concepts and skills in order to 'connect' with existing concepts that children may have. Before one can attempt to define and explain conceptual change in children, one needs to understand how learners form initial concepts regarding natural phenomena. Carey $(2009,1985)$ refers to these initial concepts as 'intuitive theories'. She defines these theories 'as theories that ground the deepest ontological commitments and the most general explanatory principles in terms of which we understand our world' (Carey 2009:26). Children attempt to make sense of the world around them from an early age by unconsciously employing basic informal empirical activities, from which they begin to reason. It is through the repetition of these activities that they form early intuitive theories. Gopnik and Wellman (2012) would argue that this process is inductive and that Bayesian theory can explain some of it. Gopnik, Meltzoff and Bryant 
(1997:11) propose that such 'conceptual structures' are similar to 'theories' of scientists. They further propose that theory formation and change form the basis of children's conceptual development and that these theories guide the child's semantic development, attributing meaning to words in language structures. According to Gopnik, Meltzhoff and Bryant (1997), children and scientists seek truths regarding the natural world by forming theories, which they then test empirically (or in mind/thought experiments, as Einstein did). Gopnik, Meltzoff and Bryant (1997) point out such similarities, saying that scientists and children form theories based on observation of the natural world and making sense of phenomena by conceptualising 'possible causes'. These observations and conceptualisations are central to the cognitive development of the child in the same way as it is central to the formation of scientific theories (Carey 2009; Carey \& Spelke 1996; Gopnik, Meltzoff and Bryant 1997). Henning (2012) explains that the similarities between the theories children form and scientific theories are that conceptualising reality includes the identification of a concept's most important characteristics, the representation of explicit and explanatory knowledge as well as the support of explanatory assumptions.

Just as scientific theories can change, based on new empirical evidence, so too can children change their theories, based on new empirical (or symbolic) knowledge about a phenomenon that can aid in making more accurate predictions and which explain the phenomenon better. According to the philosopher of science, Karl Popper (1991), a theory needs to be falsifiable for it to be regarded as a theory. Based on this view, thus, when counter-evidence is presented, contrary to the premise of the held theory, the theory needs to be adapted to include an explanation of how the counter-evidence can fit or change the existing theory. If this is not possible, the theory is abandoned and a new theory has to be developed, taking all the empirical data that are available into consideration.

In the case of children, the currently held theory need not be 'true' in the scientific sense. It merely has to make sense within the children's own conceptual systems (see Lakoff 1991; Carey 2009; Henning 2013) for an explanation of 'conceptual systems'). If new evidence is presented that does not fit the conceptual system, conceptual change has to occur in order to assimilate the new evidence. In this regard, Gopnik, Meltzoff and Bryant (1997:41) argue that the distinctive pattern of prediction, interpretation and explanation is among the best indicators of a theoretical structure and the best ways of distinguishing the theory theory from its developmental competitors'.

There are also those authors who refer to 'mental models'. Vosniadou and Brewer (1994) say that mental models are the mental representations that form when children reason about the physical world. They describe the characteristics of these mental representations as ' ... the structure is an analog to the states of the world that it represents...; ... it can be manipulated mentally ... to make predictions about the outcome of the causal states in the world...; ... and it provides explanations of physical phenomena....'. They further propose that these mental models are not static structures that are 'usually created on the spot to meet the demands of specific problemsolving situations'. In later work, Vosniadou (2009) refers to this adaptation as 'conceptual change'.

It is widely believed that intuitive theories are important building blocks in conceptual development as they serve as 'anchoring conceptions' (Clement, Brown \& Zietsman 1989) that new knowledge is built upon. Rosser (1994:170) describes intuitive theories as the 'rudimentary kernels of more sophisticated knowledge' whilst Karmiloff-Smith (1992) states that they serve as the origin for development. Spelke (1991), who argues that knowledge expands with experience, concludes that these intuitive theories are not replaced or abandoned. If the new concepts are formulated based on the intuitive theories that children hold, these intuitive theories then play an important role in learning.

It is for reasons like these that this study set out to find out what intuitive theories young children hold, regarding a number of phenomena of the natural world. This article presents data from group interviews with children, aged 6-9 years, regarding concepts from the intermediate-phase science curriculum, which they will soon encounter. The identified 'intuitive theories' will be compared qualitatively throughout the different grades so as to ascertain how children of different ages describe (express their knowledge in language) the same phenomenon. The implications of the findings for science teaching in the foundation and intermediate phase will then be discussed.

\section{Methods}

This study was conducted as a pilot study within a larger project on children's science conceptual development, to see if the 'mode of inquiry is feasible' and if it holds potential for rich data for subsequent studies. After scrutinising the foundation- and intermediate-phase curriculum, six themes were identified to serve as basis for the development of a 'science show' that would serve as the vehicle for the semistructured interviews. The science show was presented to foundation-phase children $(n=120)$ in a school in Soweto. Probing questions were asked before and after each demonstration to see what effect the experience has on the discourse of the children and to see what evidence there may be of causal language structures in their talk. There was thus a dual aim for these 'experiments': (1) finding out if the method of inquiry is feasible and (2) learning about children's causal reasoning as expressed in their talk. Added to that, the questions also attempted to identify the science content (conceptual) knowledge of the children. In some cases, children were asked to predict an outcome and evaluate their prediction after observing the demonstration.

The six themes included concepts from astronomical phenomena regarding the sun, moon and stars; the identification of 
living and nonliving objects; production of carbon dioxide gas; fire extinguishing through the release of carbon dioxide; absorption of water using paper towels and the linear motion of a paper plane propelled by an inflated balloon. The questioning technique differed with each theme. (1) The children were asked probing questions on the sections relating to astronomy and to living/ nonliving objects. (2) The production of carbon dioxide gas, extinguishing of a flame, absorption of water through a paper towel and propulsion of the paper plane were demonstrated, whilst probing questions were asked throughout the demonstration asking children to predict, describe and explain the outcomes.

Translators assisted with the interviews, probing and translating where needed when the younger children spoke in isiZulu and Sesotho. The discussions were video recorded, capturing the science show, which was presented to four classes ranging from grade $\mathrm{R}$ to grade 3 . Each class consisted of roughly 30 learners. The analysis comprised a coding of utterances that related to the topic of inquiry, specifically, whilst the rest of the data are utilised for other purposes of inquiry that are not reported in this article. After the translation from Sesotho and isiZulu to English (and backtranslated with multilingual members of the research team), the data were scrutinised for specific discourse markers about children's knowledge in the various science domains and specifically the utterances about possible causality, using the coding and categorising scheme suggested by Henning, Van Rensburg and Smit (2004; chapter 6). The instances from which one could infer causal reasoning were about $60 \%$ of total of recorded utterances.

\section{Results}

\section{Children's thoughts on science and experiments}

Before questioning the children on each of the six themes, we included a brief preamble to obtain an idea of 'the nature of science' views of the children. They were asked what they thought science was and what experiments were. Many children said that 'science is magic'. Only a few children related science to doing enquiry work. They seemed more aware of what experiments were and related experiments to 'laboratories' and work on 'animals'.

\section{Astronomical phenomena}

The questions asked in this section focused on aspects of the sun, moon and stars. Children were asked to explain the difference between night and day and to comment on the sun that was visible whilst the discussion was in progress. They were asked to describe the sun, moon and stars as objects in the sky and they had to explain what they thought these objects comprised and what their function could be. They were also asked to describe how they knew that it is morning or night. Many gave a response referring to the sun being visible in the morning. It is interesting how some responses were more indirect, referring to the temperature or colour difference between early morning and late afternoon. Some responded using contextual or social descriptors such as 'eating breakfast' or 'because the chicken crows'. There were only a few indications of causal thinking - namely, that it was night because there was no sun, and it was day because there was a sun and that the sun was a source of light.

They were also asked to describe what the sun, moon and stars consisted of and to add what the function of each was. What was evident is the change in discourse throughout the grades. The grade $\mathrm{R}$ children could not describe the sun, but instead described the function as 'gives light to see' and 'makes warm so we can play outside'. The grade 1 children said it consisted of 'light' and 'warmness', whilst the grade 2 and 3 children described the sun as a 'planet' and 'fire'. Even though these are still 'alternative' conceptions, it is noticeable that there is already conceptual change as children develop and learn.

The children from all grades described the moon as a geometric shape and knew that the moon changed its shape. The children could only identify the moon as being a 'circle' or 'banana' shaped. Only some grade 3 learners described the phases using terminology like 'new moon' or 'full moon'. The same was true for the concept of stars. The children described stars as being 'triangles and circles' - therefore, limited to representation in print material. In this instance, there was no indication of causal discourse.

The relative position of the sun and the moon proved to be challenging. The children described the position of the sun and the moon as 'up'. When asked what happened to the sun during the night, they answered by explaining that the sun 'goes away', 'goes to sleep' or 'goes home'.

\section{Living and nonliving objects}

During this section of the experimental work, we showed the children different objects, ranging from rocks and water to flowers and birds. The children were asked if the object was alive. They then had to explain 'why' they thought the object was alive or not. The characteristics children attributed to living organisms were identified after analysing their responses. The grade $\mathrm{R}$ learners only mentioned three characteristics, which included breathing, movement and ingestion of nutrients. All these were also mentioned by the children in grades 1-3 but these groups also added 'sensitivity' as a characteristic of living organisms. One child in the grade 2 group referred to excretion and some learners in grade 3 mentioned reproduction as a characteristic of living organisms. Growth was not seen as a characteristic of living organisms.

The majority classified water as nonliving with some individuals stating that water is alive because it 'moves' and because 'it is healthy'. Air and rocks were classified as nonliving objects and the explanation that most children gave was in relation to human activity or interaction. For instance, 'Air is not alive because it cannot see' because 'you 
cannot touch it [air]'. In this exercise, it was evident that children had developed the causal reasoning structure of 'it is alive because'. That they were not yet able to provide all the factual content means, simply, that they have not yet learned it.

The different age groups were largely divided when trying to classify the different parts of plants. Most children classified seeds as nonliving objects because seeds do not exhibit any of the characteristics that they associated with living organisms stating that seeds 'cannot see' or 'hear' or 'smell'. The majority of children agreed that the seed does grow and eventually becomes a plant, but did not see growth as a characteristic of life. Even after comparing their own growth to the growth of the seed and admitting that they (the children) grow and that they are alive, the seed was still not considered a living object. There were some children who mentioned that the seeds were living because 'the seed needs water to grow'. Seeds, flowers, plants and trees were mostly seen as nonliving objects.

Birds and dogs were unanimously agreed upon as being living organisms. It was much easier for the children to classify these organisms as living organisms because the animals exhibited behaviour similar to that of their own, i.e. moving, making noise with their mouths and interacting with the children. All these data had strong traces of causal thinking.

\section{Production of carbon dioxide and extinguishing a flame with carbon dioxide}

A demonstration on how to produce carbon dioxide gas using bicarbonate of soda and vinegar was carried out by placing the bicarbonate of soda into a balloon and adding vinegar. The balloon was tied and left to inflate. The children were asked to predict the outcome of the demonstration before it was conducted. The responses grew more complex when comparing the children from different grade's responses. The grade $\mathrm{R}$ children only mentioned that the balloon will inflate. All other grades also mentioned that the balloon will inflate but some children added that the balloon will pop. Thus, 'unknowingly' identifying a causal relationship between the inflation of the balloon and the capacity limits of the balloon. Some children mentioned that the reaction will 'make steam' or will 'make bubbles'. Some grade 3 learners mentioned that the balloon will 'become warm' and the balloon 'will $\mathrm{fly}^{\prime}$. The majority of learners were able to make a prediction regarding the outcome of the demonstration. Most learners could describe their observations and attempted to explain the reason for the inflation of the balloon by relating that the mixing of the reagents caused the balloon to inflate. None of the children used recognised scientific discourse when explaining the demonstration or even mentioned the production of gas but they could 'accurately predict' what the outcome would be. Another demonstration was carried out to test if they could predict what would happen if larger quantities of the reagents were added to the balloon.
Most learners could predict that the balloon would inflate more than with the previous demonstration. This shows that the children understood the relationship between cause and effect.

A second demonstration was performed using the reaction between bicarbonate of soda and vinegar. A candle was placed in a vase containing bicarbonate of soda and it was lit. The vinegar was added to the vase in order to react with the bicarbonate of soda and produce carbon dioxide that will then extinguish the flame. The children were once again asked to predict the outcome. The groups gave varying predictions with regards to what will happen to the flame ranging from 'the flame will be bigger' (grade R) or 'the flame will explode' (grade 2 and 3) to 'the flame will get wet' (grade 1) or 'the flame will get smaller' (a few grade 3 learners). None of the learners predicted that the flame would be extinguished, and after observing the demonstration, most children were astounded and proclaimed it to be 'magic'. Most children could accurately describe their observation but related the reaction to familiar examples like 'it looks like Eno's' to describe the bubbling reaction in the vase. Some grade $R$ children explained that the flame 'went out because you put the things [reagents] in there'. One grade 1 child explained that 'steam went up', which caused the flame to go out. A grade 3 learner said that the flame was extinguished because 'matches don't like water'. Once more, the children were not lacking in giving reasons, no matter what the scientific facts were.

\section{Absorption of water using paper towel and linear motion of a paper plane propelled by an inflated balloon}

Paper towel was used to demonstrate absorption by pouring water into a measuring beaker and staining the water with red food colouring. Before the paper towel was inserted into the water, the children were asked to predict the outcome. Most children predicted that the paper towel will turn red. Some learners had alternative predictions. One grade $\mathrm{R}$ and one grade 3 child said the water will turn white. Almost no children mentioned anything about a change in the water level. Only one grade 2 learner predicted that the water level will 'grow' when the towel is placed in the water. All children observed the towel turning red but did not spontaneously mention the change in water level. They only mentioned the change in the water level after being asked probing questions. Most children strugled to explain the phenomenon but some said that 'water goes into the towel because it [towel] is dry' thus indirectly comprehending a characteristic of absorbing materials. The demonstration was repeated using more towels than the previous demonstration. This time the children predicted the change in colour of the towels but also mentioned that the water level will decrease. Some grade 3 learners said that the water level will decrease more than the previous demonstration showing that they understood the relationship between adding more towels and the decrease in water level. 
The next demonstration asked the learners to predict the direction an inflated balloon will travel in if the air was let out. The balloon was attached to a paper plane which in turn was placed on a guiding piece of string to ensure that the plane moves in a linear direction. All children could correctly predict the direction that the balloon will travel in. When asked to explain the reason for this movement, some explained that the balloon moves in the opposite direction of where the opening of the balloon is. But many children thought that the movement of the plane was caused by the guiding piece of string. Once again, there was evidence that children have an urge to see a reason for phenomena or events.

\section{Discussion and conclusion: Building on children's causal frameworks}

The 'primary aim' of this study was to find some discourse pointers about children's causal reasoning. Goswami (1998:117) argues that causal reasoning plays an important part in interpreting, representing and remembering events in the natural world and adds that causal reasoning may enable children to predict and control causal events within the natural world. The ability to reason causally and to be able to express some of this logic in language could promote the development of scientific and logical reasoning in general (Legare 2014; Sobel \& Legare 2014).

The ability of the children to reason causally was evident in the sample group participating in this study. In all instances, children were able to explain and predict possible outcomes of the demonstration, although not scientifically accurate. They engaged in talk on the two topics of living organisms and the theme of the sun, the moon and the stars with a strong urge to explain and reason, despite their lack of factual knowledge. In the introduction to this article, I emphasised that children may need (learned) knowledge to act upon the 'placeholders' of causality and of language that they already have (Carey 2009). This shows that children in the foundation phase not only have the ability to recognise causality but they are also able to use this ability incrementally as they gain more knowledge - as the difference between the age groups (and their accompanying learning) shows in this sample of qualitative data. What is lacking, however, is sound scientific concepts, most likely because of the sparse inclusion of science in the foundation-phase curriculum. This is also the view of Saçkes (2014) in his study of a theoretical model of teaching science for young children.

As early as 1982, Gilbert, Osborne and Fensham described the intuitive theories of children as 'children's science', and they identified some patterns in 'children's science'. These included that children use 'everyday language' when describing phenomena that are incoherent with the accepted scientific definition; they have a self-centred and human-centred viewpoint of phenomena; they believe non-observables do not exist; they endow objects with the characteristics of humans and animals and also endow objects with a certain amount of a physical quantity. Some of these patterns were also identified in this study. The language that the children in this study used seldom fitted the registers of science. Most of the children attributed human qualities to their naïve classification of living organisms and could not fathom that plants were living organisms, as their characteristics are so far removed or even alien' to that which the children considered to be acceptable in their framework. I would argue that their understanding can increase with only some systematic teaching, aiming to let them reason inductively from (live) examples around them in classroom plants, something they are prone to do, if guided, quite easily, according to Gopnik (2011).

This study set out to find out if children are capable of reasoning causally by identifying their intuitive theories regarding certain scientific phenomena. The importance of the findings of this study lies in the identification of possible intuitive theories of children that teachers could utilise when formal science teaching starts in the intermediate phase. This study also shows that children in the foundation phase of schooling are indeed capable of predicting outcomes of scientific demonstrations and explaining the causes of these outcomes even though their predictions or explanations are not scientifically correct. These are fundamental skills in the learning of science concepts and are essential for critical thinking in science. The data confirm the notion that children do not enter the classroom as blank slates but hold many intuitive theories regarding scientific phenomena and that they are able to see causality, even when lacking detail.

When trying to describe phenomena, children not only use observable evidence to explain a phenomenon but also call on social and contextual memories to describe these phenomena. When describing the shape of the moon or stars, they refer to geometrical shapes instead of using the scientific discourse. A possible reason for this can be found in the teaching of how to draw the moon and stars. Some teachers use geometric shapes like circles and triangles to teach children to draw the moon and stars. Children are then taught to associate the scientific phenomenon with concepts from other fields where these concepts impose on the nuances of the scientific phenomena.

Differentiating between living and nonliving objects is critical to the development of a child's concept of biology and essential for all biological thought (Slaughter, Jaakkola \& Carey 1999; Siegal \& Peterson 1999; Springer 1999). Venville (2004) argues that:

children who are able to consistently recognize life, as biologists do, are in an appropriate cognitive position to develop new concepts and beliefs that are commensurate with core biological theory.

Carey (1985) found that children first compare structures and behaviour of animals to those of humans. They then realise the common structures and behaviour between humans and 
animals, and they then 'make an inductive from people to animals based on the perceptual similarities of the animal to human' (Venville 2004). Carey notes that children only realise that plants portray characteristics of living organisms by the age of 10 or 11 . I would add, although, that if they learn some of the formal structures, in other words the linguistic descriptors, they may be somewhat better prepared for ultimate understanding. I again refer to Vygotsky (1986) and Vygotsky and Luria (1994) who make this argument language and concepts meet each other and it is not the same for all children.

Many children in this study did not mention growth, reproduction or excretion as characteristics to classify an object as a living object but used movement, sensitivity and metabolism (breathing) as characteristics to classify an object as living. These children may not have been exposed to enough examples of different animals, because they do not see many animals around them in everyday life and therefore do not see animals that excrete or reproduce on a regular basis. For children who live in farming areas, the case may be different. For children in Soweto, where pets are not the norm for households, there are few opportunities to see animal life in their everyday setting. The same accounts for children who do not encounter plant life. These children did not classify plants as living organisms because they encounter them when they are sold as vegetables, fruit or flowers, but do not, for example, look after their own little garden as children. Some children have, for example, never seen the roots of a plant and find it a strange phenomenon. There is, however, another possible explanation: they regard themselves as the primary example of a living organism and plants do not show signs of life like animals do, especially mammals, like humans. Pine, Messer and St. John (2001) found that UK teachers say children think that plants and trees cannot be living organisms because they do not move and their concept of living things is that it must move. It would be interesting to compare this study with a similar one conducted with children living in a rural environment where they are exposed to farming with animals and growth of crops from a young age.

Legare and Clegg (2015) argue that the repetition of consistent outcomes of phenomena provide an important opportunity for children to deepen their understanding of phenomena and will allow them to produce causal mechanisms. Legare (2014) found that there is even more importance in exposing children to events that are 'inconsistent with their current explanations'. These inconsistencies are more noteworthy and informative to the children as they can formulate new hypotheses adapted from their prior beliefs. Using this information, teacher's will be able to adapt their teaching to include contrastive outcomes and counterfactuals to ensure that children are more engaged during demonstrations.

This study showed that the participating children in the foundation phase are capable of reasoning causally but lack the scientific content that they need to 'populate' their causal reasoning ability. I believe that teaching more science concepts in the earlier grades will only be to the benefit of young learners. Further to that, if science can be integrated with mathematics and language teaching, the mathematical and the linguistic components of causal reasoning may be strengthened even more.

The 'secondary aim' of this investigation was one of methodology. I wanted to find out if this type of approach to research on children's reasoning and conceptual development is usable. The sample was not big, but it would seem that the live 'science show' elicited direct experiential comments. The questions asked prior to the science show produced far less usable data. The method can be used in a larger study, fine-tuning some of the aspects. The translations worked quite well too. In the next round, we will also use backtranslations and ask the children if we have 'heard' them correctly.

\section{Acknowledgements}

This study was conducted as part of the National Research Foundation grant 90372.

\section{Competing interests}

The author declares that he has no financial or personal relationships that may have inappropriately influenced him in writing this article.

\section{References}

Bowerman, M. \& Levinson, S.C., 2001, Language acquisition and conceptual development, Vol. 3, Cambridge University Press, Cambridge.

Brown, J.S., Collins, A. \& Duguid, P., 1989, 'Situated cognition and the culture of learning', Educational Researcher 18(1), 32-42.

Carey, S., 1985, Conceptual change in childhood, Bradford Books, MIT Press, Cambridge.

Carey, S., 2009, The origin of concepts, Oxford University Press, Oxford.

Carey, S. \& Spelke, E., 1996, 'Science and core knowledge', Philosophy of Science 63(4), 515-533.

Clement, J., Brown, D.E. \& Zietsman, A., 1989, 'Not all preconceptions are misconceptions: Finding "anchoring conceptions" for grounding instruction on students' intuitions', International Journal of Science Education 11(5), 554-565.

Cockcroft, 2015

Department of Basic Education, 2011.

Furner, J.M. \& Kumar, D.D., 2007, 'The mathematics and science integration argument: A stand for teacher education', Eurasia Journal of Mathematics, Science \& Technology Education 3(3), 185-189.

Gilbert, J.K., Osborne, R.J. \& Fensham, P.J., 1982, 'Children's science and its consequences for teaching', Science Education 66(4), 623-633.

Gopnik, A., 2003, 'The theory as an alternative to the innateness hypothesis', Chomsky and His Critics, 238-254.

Gopnik, A., 2011.

Gopnik, A., Meltzoff, A.N. \& Bryant, P., 1997, Words, thoughts, and theories, vol. 1 MIT Press, Cambridge.

Gopnik, A., Sobel, D.M., Schulz, L.E. \& Glymour, C., 2001, 'Causal learning mechanisms in very young children: Two-, three-, and four-year-olds infer causal relations from patterns of variation and covariation', Developmental Psychology 37(5), 620.

Gopnik, A. \& Wellman, H.M., 2012, 'Reconstructing constructivism: Causal models, Bayesian learning mechanisms, and the theory', Psychological Bulletin 138(6), 1085.

Goswami, U., 1998, Cognition in Children, Psychology Press, Abingdon.

Henning, E., 2012, 'Learning concepts, language, and literacy in hybrid linguistic codes: The multilingual maze of urban grade 1 classrooms in South Africa', Perspectives in Education 30(3), 69-77. 
Henning, E., 2013, 'Teachers' understanding of mathematical cognition in childhood: Towards a shift in pedagogical content knowledge?' Perspectives in Education 31(3), 139-154.

Henning, E. \& Dampier, G., 2012, 'Linguistic liminality in the early years of school Urban South African children 'betwixt and between' languages of learning', South African Journal of Childhood Education 2(1), 101-120.

Henning, E. \& Ragpot, L., 2015, 'Pre-school children's bridge to symbolic knowledge: First framework for a cognition lab at a South African university', South African Journal of Psychology 45(1), 71-80.

Henning, E., Van Rensburg, W. \& Smit, B., 2004, Finding your way in qualitative research, Van Schaik, Pretoria.

Karmiloff-Smith, A., 1992, Beyond modularity: A developmental perspective on cognitive science, MIT Press, Cambridge.

Klaar, S. \& Öhman, J., 2014, 'Doing, knowing, caring and feeling: Exploring relations between nature-oriented teaching and preschool children's learning', International Journal of Early Years Education 22(1), 37-58.

Lakoff, G., 1991, 'Cognitive versus generative linguistics: How commitments influence results', Language \& Communication 11(1), 53-62.

Lave, J., 1988, Cognition in practice: Mind, mathematics and culture in everyday life, Cambridge University Press, Cambridge.

Legare, C.H., 2014, 'The contributions of explanation and exploration to children's scientific reasoning', Child Development Perspectives 8(2), 101-106.

Legare, C.H. \& Clegg, J.M., 2015, in S. Robson \& S. Quinn (eds.), Routledge internationa handbook on young children's thinking and understanding, pp. 65-74, Routledge, London.

Passy, R., 2014, 'School gardens: Teaching and learning outside the front door', Education 3-13 42(1), 23-38.

Piaget, J., 1952, The origins of intelligence in children, Vol. 8, No. 5, p. 18, International Universities Press, New York.

Piaget, J. \& Inhelder, B., 1969, The psychology of the child, Basic Books, New York.

Piaget, J. \& Inhelder, B., 1975, The origin of the idea of chance in children, transl. L. Leake, P. Burrell \& H.D. Fishbein, WW Norton, New York.

Piaget, J., Inhelder, B. \& Häfliger, L., 1977, Die Psychologie des Kindes, Fischer, Frankfurt.

Pine, K., Messer, D. \& St. John, K., 2001, ‘Children's misconceptions in primary science: A survey of teachers' views', Research in Science \& Technological Education 19(1), 79-96. http://dx.doi.org/10.1080/02635140120046240

Popper, K., 1991, In search of a better world, Blackwell, Oxford.
Rogoff, B., 1990, Apprenticeship in thinking: Cognitive development in social context, Oxford University Press, Oxford

Rosser, R.A., 1994, Cognitive development: Psychological and biological perspectives, Allyn \& Bacon, Boston.

Saçkes, M., 2014, 'How often do early childhood teachers teach science concepts? Determinants of the frequency of science teaching in kindergarten', European Early Childhood Education Research Journal 22(2), 169-184.

Siegal, G. \& Peterson, C.C., 1999, 'Becoming mindful of biology and health: An introduction', in M. Siegal \& C. Peterson (eds.), Children's understanding of biology and health, pp. 1-19, Cambridge University Press, Cambridge.

Slaughter, V., Jaakkola, R. \& Carey, S., 1999, 'Constructing a coherent theory: Children's biological understanding of life and death', in M. Siegal \& C. Peterson (eds.), Children's understanding of biology and health, pp. 71-96, Cambridge University Press, Cambridge.

Sobel, D.M. \& Legare, C.H., 2014, 'Causal learning in children', Wiley Interdisciplinary Reviews: Cognitive Science 5(4), 413-427.

Spelke, E.S., 1991, 'Physical knowledge in infancy: Reflections on Piaget's theory', The Epigenesis of Mind: Essays on Biology and Cognition 133-169.

Spelke, E.S., 2010, 'Innateness, choice, and language', in J. Bricmont \& J. Franck (eds.), Chomsky notebook, pp. 203-210, Columbia University Press, New York. [Originally published in French: Spelke, E.S., 2007., 'Innéisme, liberté et language', in J. Bricmont \& J. Franck (eds.), Cahier no 88: Noam Chomsky, pp. 197-201, L’Herne, Paris.]

Springer, K., 1999, 'How a naïve theory of biology is acquired', in M. Siegal \& C. Peterson (eds.), Children's understanding of biology and health, pp. 45-70, Cambridge University Press, Cambridge.

Venville, G., 2004, 'Young children learning about living things: A case study of conceptual change from ontological and social perspectives', Journal of Research in Science Teaching 41(5), 449-480.

Vosniadou, S. (ed.), 2009, International handbook of research on conceptual change, Routledge, London.

Vosniadou, S. \& Brewer, W.F., 1994, 'Mental models of the day/night cycle', Cognitive Science 18(1), 123-183.

Vosniadou, S. \& loannides, C., 1998, 'From conceptual development to science education: A psychological point of view', International Journal of Science Education 20(10), 1998.

Vygotsky, L.S., 1978, 'Interaction between learning and development', Readings on the Development of Children, 23(3), 34-41.

Vygotsky, L.S., 1986, Thought and language, transl. A. Kozulin, MIT Press, Cambridge.

Vygotsky, L.S. \& Luria, A., 1994, 'Tool and symbol in child development', The Vygotsky Reader 99-174. 